\title{
MULTIDIMENSIONAL EXPONENTIAL TIMESTEPPING WITH BOUNDARY TEST*
}

\author{
KALVIS M. JANSONS ${ }^{\dagger}$ AND G. D. LYTHE
}

\begin{abstract}
Exponential timestepping algorithms are efficient for exit-time problems because a boundary test can be performed at the end of each timestep, giving high-order convergence in numerical evaluation of mean exit times. Successive time increments are independent random variables with an exponential distribution. We show how to perform exact timestepping for Brownian motion in more than one dimension and consider hitting times of curved surfaces. Taking as examples the exit times from a circle, from a ball, from an ellipse, and from the region bounded by the two lines of a hyperbola, we report the results of numerical experiments performed with the algorithms developed in this work.
\end{abstract}

Key words. stochastic calculus, numerical methods, multidimensional diffusion, exit time, curved boundaries

AMS subject classifications. $60-08,65 \mathrm{C} 30$

DOI. $10.1137 / 040612865$

1. Introduction. One numerical realization of a stochastic process is a series of values generated successively. Under standard algorithms [1], the values correspond to times separated by a fixed, or at least deterministic, duration $\Delta t$. Under exponential timestepping [2], the times corresponding to numerical values are instead separated by independent realizations of a random variable $\delta \boldsymbol{t}$, where

$$
\mathcal{P}[\boldsymbol{\delta} \boldsymbol{t}>t]=\exp (-\lambda t)
$$

and $\lambda$ is a constant. Thus $\langle\boldsymbol{\delta} \boldsymbol{t}\rangle=\lambda^{-1}$, where angled brackets denote mean over realizations. Random variables are set in boldface.

The increment of Brownian motion in one dimension with diffusivity $D$ over a fixed time interval $\Delta t$ is a Gaussian random variable with mean zero and variance $2 D \Delta t$. Thus exact numerical updates require realizations of Gaussian random variables. If the time interval is not fixed but distributed according to (1.1), the increment of Brownian motion has a symmetric exponential distribution [2]. Exact updates under exponential timestepping therefore require realizations of random variables with an exponential distribution, which are easily generated as $-\log \mathbf{u}$, where $\mathbf{u}$ is distributed uniformly on $(0,1)$. In this work, we construct the exact update for Brownian motion in $n$ dimensions by separating the increment into a modulus and an angle.

The particular numerical problem we focus on in this paper is the measurement of exit or passage times, where the quantity of interest is the first time that a stochastic process attains a given value or exits a region $[3,4,5,6,7,8]$. A numerical scheme for such a task has three components. The first component is an algorithm for updating the stochastic process so that realizations can be generated. The second is a criterion for deciding when the path hits the boundary, bringing the realization to an end. The

*Received by the editors August 4, 2004; accepted for publication (in revised form) May 17, 2005; published electronically November 15, 2005.

http://www.siam.org/journals/sisc/27-3/61286.html

${ }^{\dagger}$ Department of Mathematics, University College London, Gower Street, London WC1E 6BT, England (multidim@randomideas.org, http://randomideas.org).

${ }^{\ddagger}$ Department of Applied Mathematics, University of Leeds, Leeds LS2 9JT, England (expmultidim @randomideas.org, http://randomideas.org). 
third is a relationship between the mean number of numerical timesteps per realization and the mean exit time.

The simplest criterion for bringing a realization to an end is that the process be outside the region at the end of a timestep. Numerical schemes using this criterion overestimate the mean exit time because of the strictly positive probability that an excursion is made outside the region during a timestep that starts and ends inside the region $[2,9,10,11,12,13,14,15]$. To counteract this systematic error, a boundary test can be performed after each timestep, in which with probability $p_{i}$ the realization is brought to an end, where $p_{i}$ is an estimate for the conditional probability, given the starting and ending positions of a timestep, that an excursion out of the region has been made during the timestep $[2,10,11,12,13,14,15]$. Such tests have been shown to reduce the systematic error in mean exit times from being proportional to $\Delta t^{1 / 2}$ to being proportional to $\Delta t$ when $\Delta t$ is fixed [13]. Under exponential timestepping, the corresponding improvement is from an error proportional to $\langle\boldsymbol{\delta} \boldsymbol{t}\rangle^{1 / 2}$, i.e., to the square root of the mean duration of a timestep, to an error proportional to $\langle\boldsymbol{\delta} \boldsymbol{t}\rangle[2]$. Alternatively, under the walk-on-spheres method, a path is constructed as a sequence of jumps from ball centers to ball surfaces and an absorption layer is defined close to the boundary $[16,17]$. Realizations are brought to an end when they enter the layer.

The advantage of exponential timestepping is that expressions for the density of increments and for the conditional hitting probabilities required to devise boundary tests have a simple form. They are the Laplace transform of the corresponding quantities under fixed-duration timesteps and can be derived intuitively using the equivalence between the end of an exponential timestep and a mark on the path made by an independent Poisson point process with constant rate. In this work, we devise boundary tests under exponential timestepping in more than one dimension. Flat boundaries are equivalent to the one-dimensional case and thus can be dealt with exactly. Approximate boundary tests for smooth boundaries are derived and are valid when the radius of curvature is much larger than typical displacements in one timestep.

Armed with a criterion for bringing realizations to a stop, we generate large numbers of them and estimate the mean exit time from the mean number of timesteps per realization. In the case of timesteps of fixed duration $\Delta t$, the elapsed time until the end of the timestep at which the boundary was deemed hit is known exactly. The time during the last timestep at which the boundary is hit is not known and must be estimated to obtain a mean exit time accurate to better than $\Delta t$ [18]. In the case of exponential timestepping, the duration of any one timestep is not generated or known. Despite this apparent complication, evaluation of the mean exit time is straightforward under exponential timestepping because an update can be thought of as a jump to the next mark on the Brownian path made by an independent Poisson point process with rate $\lambda$ (Figure 1.1). If $N$ is the mean number of marks before a random time $\mathbf{H}$ that is independent of the marking process, then

$$
\langle\mathbf{H}\rangle=\lambda N,
$$

whatever the distribution of $\mathbf{H}$. Suppose there are $M$ realizations and the boundary is deemed to have been hit during the $n_{j}$ th timestep of realization $j$. The numerical estimate of the mean exit time is $M^{-1} \sum_{j=1}^{M}\left(n_{j}-1\right)$.

Section 2 is a summary of basic results in one dimension and multiple dimensions. In section 3, we consider hitting times of curved boundaries. Exponential timestepping methods in $n$ dimensions are described in section 4 . Section 5 contains the results 


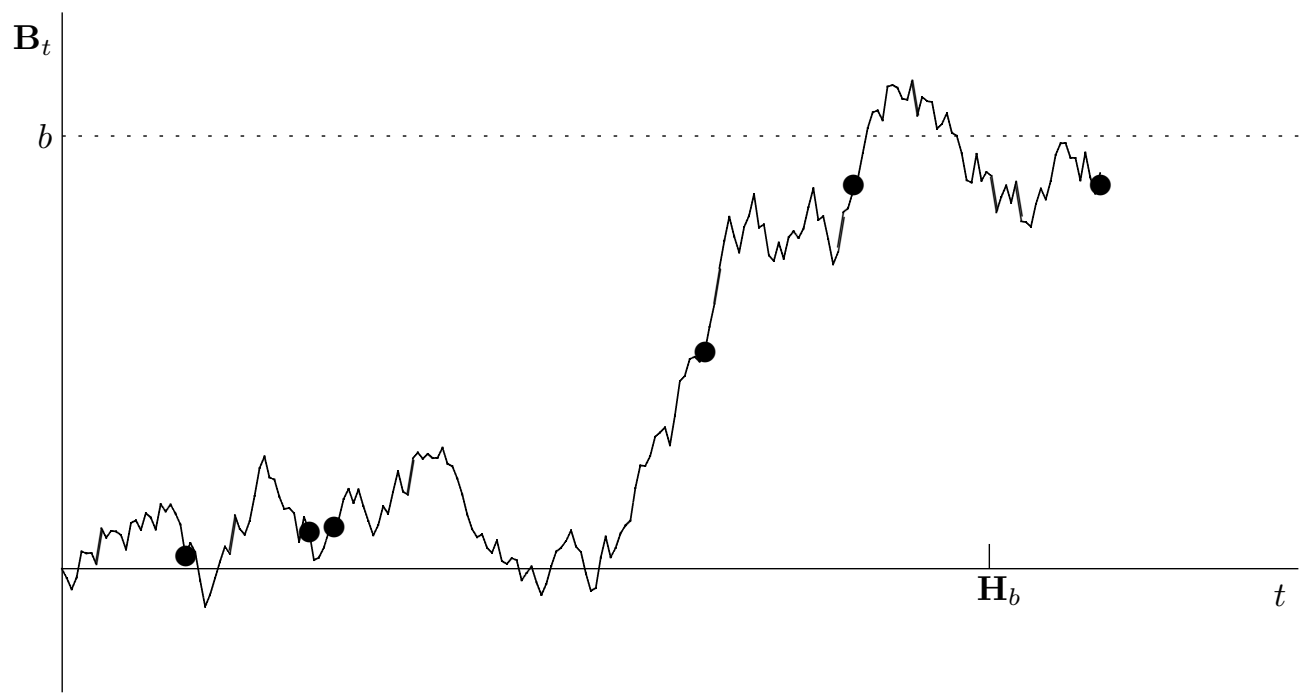

FIG. 1.1. One realization is depicted. Exponential timesteps are jumps from one black point to the next. The values of the process at these times, but not the times themselves, are generated. The aim is to estimate the mean of $\mathbf{H}_{b}$ from many realizations.

of numerical experiments with the new algorithms described here and with the Euler algorithm.

2. Basic results and notation. An $n$-dimensional Brownian motion $\mathbf{B}$ with diffusivity $D$ has Cartesian components that are independent Brownian motions with diffusivity $D . \mathbf{B}_{0}=0$ and the probability density function of $\mathbf{B}_{t}$ for any fixed $t>0$ is

$$
p^{(n)}(x, t)=(4 \pi D t)^{-n / 2} \exp \left(-|x|^{2} / 4 D t\right),
$$

where $x=\left(x_{1}, x_{2}, \ldots, x_{n}\right)$ and $|x|^{2}=\sum_{i=1}^{n} x_{i}^{2}$. Consider an exponential timestep, started at 0 , with mean duration $\lambda^{-1}$. The density of $\mathbf{B}_{\delta t}$ is [23]

$$
\begin{aligned}
p_{\nu}^{(n)}(x) & =\int_{0}^{\infty} \lambda \exp (-\lambda t) p^{(n)}(x, t) \mathrm{d} t \\
& =\frac{\nu^{n}}{2 \pi}(2 \pi \nu|x|)^{1-n / 2} K_{n / 2-1}(\nu|x|),
\end{aligned}
$$

where $K_{m}$ is the modified Bessel function of the second kind of order $m$ and

$$
\nu=(\lambda / D)^{1 / 2} .
$$

In one dimension, let $\mathbf{H}_{b}=\inf \left\{t>0: \mathbf{B}_{t}=b\right\}$ and let $u(t)=\frac{\mathrm{d}}{\mathrm{d} t} \mathcal{P}\left[\mathbf{H}_{b}<t\right]$. Then $[3,7,2]$

$$
u(t)=|b|\left(4 \pi D t^{3}\right)^{-1 / 2} \exp \left(-b^{2} / 4 D t\right) .
$$

The probability that $\mathbf{H}_{b}<\boldsymbol{\delta} \boldsymbol{t}$ is given by

$$
\mathcal{P}\left[\mathbf{H}_{b}<\boldsymbol{\delta} \boldsymbol{t}\right]=\mathrm{e}^{-\nu|b|} .
$$


If the value at the end of the timestep is known, we have the following conditional probability:

$$
\mathcal{P}\left[\mathbf{H}_{b}<\boldsymbol{\delta} \boldsymbol{t} \mid \mathbf{B}_{\boldsymbol{\delta} t}=z\right]=\mathrm{e}^{-2 \nu|b-\tilde{z}|},
$$

where $\tilde{z}$ is the closer of 0 and $z$ to $b$. The one-dimensional results (2.5) and (2.6) hold for flat surfaces in any number of dimensions. For curved surfaces it remains true that the probability of hitting a surface during a timestep is significant when the starting or ending value is within a layer of width $\nu^{-1}$ of the surface.

2.1. Hitting circles and spheres. Exact results are also known for exit times from $n$-dimensional balls. Let

$$
\mathbf{h}_{R}^{(n)}(r)=\inf \left\{t>0:\left|\mathbf{B}_{t}\right|=R|| \mathbf{B}_{0} \mid=r\right\},
$$

the first time $t>0$ that $\left|\mathbf{B}_{t}\right|=R$, starting from $\left|\mathbf{B}_{0}\right|=r$. Then $[8,19]$

$$
\mathcal{P}\left[\mathbf{h}_{R}^{(n)}(r)<\boldsymbol{\delta} \boldsymbol{t}\right]= \begin{cases}\left(\frac{r}{R}\right)^{1-n / 2} \frac{I_{n / 2-1}(\nu r)}{I_{n / 2-1}(\nu R)}, & r<R, \\ \left(\frac{r}{R}\right)^{1-n / 2} \frac{K_{n / 2-1}(\nu r)}{K_{n / 2-1}(\nu R)}, & r \geq R,\end{cases}
$$

where $I_{m}$ is the modified Bessel function of the second kind of order $m$.

Now consider the density of increments of the modulus of Brownian motion in $n$ dimensions. Define

$$
b^{(n)}\left(r, r_{0}\right)=\frac{\mathrm{d}}{\mathrm{d} r} \mathcal{P}\left[\left|\mathbf{B}_{\delta t}\right|<r|| \mathbf{B}_{0} \mid=r_{0}\right] .
$$

Then [19]

$$
b^{(n)}\left(r, r_{0}\right)= \begin{cases}\nu^{2} r^{n / 2} r_{0}^{-n / 2+1} I_{n / 2-1}\left(\nu r_{0}\right) K_{n / 2-1}(\nu r), & r_{0}<r, \\ \nu^{2} r^{n / 2} r_{0}^{-n / 2+1} K_{n / 2-1}\left(\nu r_{0}\right) I_{n / 2-1}(\nu r), & r_{0} \geq r .\end{cases}
$$

An advantage of the exponential duration of a timestep is that many conditional probabilities factorize. In particular, using (2.8) and (2.10) gives

$$
\begin{array}{r}
\mathcal{P}\left[\mathbf{h}_{R}^{(n)}\left(r_{0}\right)<\boldsymbol{\delta} \boldsymbol{t}|| \mathbf{B}_{\delta \boldsymbol{t}} \mid=r_{1}\right]=\mathcal{P}\left[\mathbf{h}_{R}^{(n)}\left(r_{0}\right)<\boldsymbol{\delta} \boldsymbol{t}\right]\left(b^{(n)}\left(r_{1}, r_{0}\right)\right)^{-1} b^{(n)}\left(r_{1}, R\right) \\
= \begin{cases}\frac{I_{n / 2-1}\left(\nu\left(r_{0} \vee r_{1}\right)\right)}{I_{n / 2-1}(\nu R)} \frac{K_{n / 2-1}(\nu R)}{K_{n / 2-1}\left(\nu\left(r_{0} \vee r_{1}\right)\right)}, & \left(r_{0} \vee r_{1}\right)<R, \\
\frac{I_{n / 2-1}(\nu R)}{I_{n / 2-1}\left(\nu\left(r_{0} \wedge r_{1}\right)\right)} \frac{K_{n / 2-1}\left(\nu\left(r_{0} \wedge r_{1}\right)\right)}{K_{n / 2-1}(\nu R)}, & \left(r_{1} \wedge r_{0}\right) \geq R,\end{cases}
\end{array}
$$

where $(a \vee b)=\max (a, b)$ and $(a \wedge b)=\min (a, b)$.

It is illuminating to compare these results to (2.5) and (2.6). Taking the limit of (2.8) as $\nu R \rightarrow \infty$ with $S=\nu(R-r)$ fixed gives

$$
\mathcal{P}\left[\mathbf{h}_{R}^{(n)}(r)<\boldsymbol{\delta} \boldsymbol{t}\right]=\mathrm{e}^{-|S|}\left(1-\frac{1}{2}(n-1)(\nu R)^{-1} S+\mathcal{O}\left((\nu R)^{-2}\right)\right) .
$$




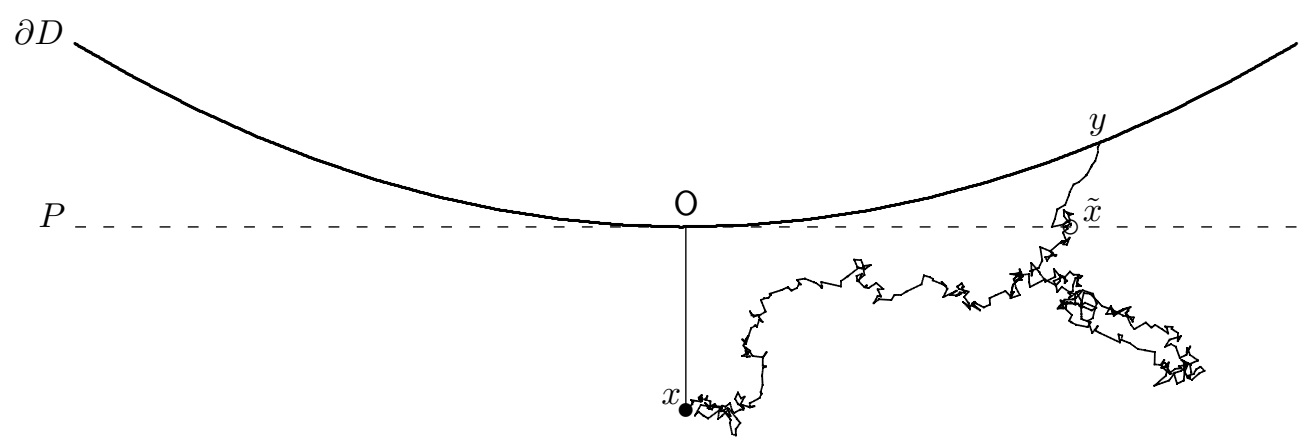

FIG. 3.1. The initial position $x$ is marked with a filled circle. Before hitting the curved surface $\partial D$, the path of the stochastic process hits the surface $P$, tangent to the point on $\partial D$ closest to $x$. The distance from $x$ to $\mathrm{O}$ is denoted by $s$.

In (2.11), let $r$ be the closer of $r_{0}$ and $r_{1}$ to $R$. In the same limit,

$$
\mathcal{P}\left[\mathbf{h}_{R}^{(n)}\left(r_{0}\right)<\boldsymbol{\delta} \boldsymbol{t}|| \mathbf{B}_{\delta \boldsymbol{t}} \mid=r_{1}\right]=\exp (-2|S|)\left(1-\frac{n^{2}-4 n+3}{(2 \nu R)^{2}} S+\mathcal{O}\left((\nu R)^{3}\right)\right) .
$$

3. Curved boundaries. Consider a timestep started at a point $x$ in a concave domain in $\mathbb{R}^{n}$ with boundary $\partial D$ (Figure 3.1 ). We place $\mathrm{O}$, the origin of coordinates in $\mathbb{R}^{n}$, at the point on $\partial D$ nearest to $x$; define the $x_{n}$ direction to be along the line $x \mathrm{O}$; and let $s$ denote the distance from $x$ to $\mathrm{O}$. Let $P$ be the $(n-1)$-dimensional surface tangent to $\partial D$ at $\mathrm{O}$. Let $\mathbf{H}_{P}^{(n)}(x)$ be the first hitting time of $P$,

$$
\mathbf{H}_{P}^{(n)}(x)=\inf \left\{t>0: \mathbf{B}_{t} \in P \mid \mathbf{B}_{0}=x\right\} .
$$

We denote the value of $\mathbf{B}$ at the time $\mathbf{H}_{P}^{(n)}(x)$ by $\tilde{x}$ and write $\tilde{x}=\left(\tilde{x}^{\perp}, 0\right)$ where $\tilde{x}^{\perp}=\left(\tilde{x}_{1}, \tilde{x}_{2}, \ldots, \tilde{x}_{n-1}\right)$.

Independent of $n$, we have

$$
\mathcal{P}\left[\mathbf{H}_{P}^{(n)}(x)<\boldsymbol{\delta} \boldsymbol{t}\right]=\mathrm{e}^{-\nu s} .
$$

Consider the subset of paths for which $\mathbf{H}_{P}^{(n)}(x)<\boldsymbol{\delta} \boldsymbol{t}$. For an event $\Theta$, let

$$
\mathcal{S}[\Theta]=\mathcal{P}\left[\Theta \mid \mathbf{H}_{P}^{(n)}(x)<\boldsymbol{\delta} \boldsymbol{t}\right]=\frac{\mathcal{P}\left[\Theta, \mathbf{H}_{P}^{(n)}(x)<\boldsymbol{\delta} \boldsymbol{t}\right]}{\mathcal{P}\left[\mathbf{H}_{P}^{(n)}(x)<\boldsymbol{\delta} \boldsymbol{t}\right]} .
$$

Let $q_{P}\left(x^{\perp}\right)$ be the density of arrival positions on the surface $P$, i.e.,

$$
q_{P}\left(x^{\perp}\right)=\frac{\partial}{\partial x_{1}} \frac{\partial}{\partial x_{2}} \cdots \frac{\partial}{\partial x_{n-1}} \mathcal{S}\left[\tilde{x}_{1}<x_{1}, \tilde{x}_{2}<x_{2}, \ldots, \tilde{x}_{n-1}<x_{n-1}\right] .
$$

The density of arrival times on $P$, conditioned on being earlier than $\boldsymbol{\delta} \boldsymbol{t}$, is

$$
u_{\nu}(t)=\frac{\mathrm{d}}{\mathrm{d} t} \mathcal{S}\left[\mathbf{H}_{P}^{(n)}(x)<t\right] .
$$


Using (2.4), we find that

$$
u_{\nu}(t)=s \exp (\nu s)\left(2 \pi t^{3}\right)^{-1 / 2} \exp \left(-s^{2} / 2 t-\nu^{2} t / 2\right) .
$$

An expression for $q_{P}\left(x^{\perp}\right)$ is obtained by integrating $u_{\nu}(t)$ over the density of an $(n-1)$-dimensional Brownian motion. Explicitly,

$$
\begin{aligned}
q_{P}\left(x^{\perp}\right) & =\int_{0}^{\infty} u_{\nu}(t)(2 \pi t)^{-(n-1) / 2} \exp \left(\frac{-\left|x^{\perp}\right|^{2}}{2 t}\right) \mathrm{d} t \\
& =2 s \exp (\nu s)\left(\frac{\nu}{2 \pi}\right)^{n / 2}\left(s^{2}+\left|x^{\perp}\right|^{2}\right)^{-n / 4} K_{n / 2}\left(\nu\left(s^{2}+\left|x^{\perp}\right|^{2}\right)^{1 / 2}\right) .
\end{aligned}
$$

Note that, for $1 \leq i \leq n-1$,

$$
\int x_{i}^{2} q_{P}\left(x^{\perp}\right) \mathrm{d} x^{\perp}=\int_{-\infty}^{\infty} x^{2}\left(s^{2}+x^{2}\right)^{-1 / 4} K_{1}\left(\nu\left(s^{2}+x^{2}\right)^{1 / 2}\right) \mathrm{d} x=\frac{s}{\nu} .
$$

Timesteps during which the boundary is hit typically have $\nu s=\mathcal{O}(1)$. Thus the typical spread of hitting points on $P$ is such that $\tilde{x}_{i}=\mathcal{O}\left(\nu^{-1}\right)$.

Now we consider the probability of hitting the curved boundary $\partial D$. Similarly to (3.1), let

$$
\mathbf{H}_{\partial D}^{(n)}(x)=\inf \left\{t>0: \mathbf{B}_{t} \in \partial D \mid \mathbf{B}_{0}=x\right\} .
$$

For the geometry chosen for Figure $3.1, \mathcal{P}\left[\mathbf{H}_{\partial D}^{(n)}(x)<\boldsymbol{\delta} \boldsymbol{t}\right]<\mathcal{P}\left[\mathbf{H}_{P}^{(n)}(x)<\boldsymbol{\delta} \boldsymbol{t}\right]$. We invoke the following "memoryless" property of exponential timesteps: the conditional probability of hitting $\partial D$ before the end of a timestep, given that a point $\tilde{x} \in P$ is hit during the timestep, is equal to the probability of hitting $\partial D$ during a timestep started at $\tilde{x}$. Thus

$$
\mathcal{P}\left[\mathbf{H}_{\partial D}^{(n)}(x)<\boldsymbol{\delta} \boldsymbol{t}\right]=\mathrm{e}^{-\nu s} \int q_{P}\left(\tilde{x}^{\perp}\right) \mathcal{P}\left[\mathbf{H}_{\partial D}^{(n)}(\tilde{x})<\boldsymbol{\delta} \boldsymbol{t}\right] \mathrm{d} \tilde{x}^{\perp} .
$$

When the boundary is flat, the integral in (3.11) is unity and we regain (3.2). As $\nu \rightarrow \infty$ with $\nu s$ fixed, a smooth surface appears almost flat on the scale of typical displacements in one timestep and there is a small correction to (3.2).

Given a point $y$ on $\partial D$, let $y=\left(y^{\perp}, y_{n}\right)$, where $y^{\perp}=\left(y_{1}, y_{2}, \ldots, y_{n-1}\right)$. The directions of the axes are chosen so that the surface $\partial D$ is parametrized as $y_{n}=$ $y_{n}\left(y^{\perp}\right)$, where $y_{n}\left(y^{\perp}\right)$ has the Taylor series [21]

$$
y_{n}\left(y^{\perp}\right)=\frac{1}{2}\left(\kappa_{1} y_{1}^{2}+\kappa_{2} y_{2}^{2}+\cdots+\kappa_{n-1} y_{n-1}^{2}\right)+\cdots .
$$

Let $\kappa=\max _{i} \kappa_{i}$. The statement that $\nu$ is sufficiently large so that the maximum radius of curvature of $\partial D$ is smaller than typical displacements in one timestep is equivalent to taking $\kappa / \nu \ll 1$.

The probability (3.2) is the lowest-order estimate of $\mathcal{P}\left[\mathbf{H}_{\partial D}^{(n)}(x)<\boldsymbol{\delta} \boldsymbol{t}\right]$, the probability of hitting a curved boundary $\partial D$ before the end of a timestep started at $x$. At next order, $\mathcal{P}\left[\mathbf{H}_{\partial D}^{(n)}(\tilde{x})<\boldsymbol{\delta} \boldsymbol{t}\right]$ in $(3.11)$ is approximated by the probability of hitting the tangent plane through the point on $\partial D$ closest to $\tilde{x}$ [22]. That is,

$$
\mathcal{P}\left[\mathbf{H}_{\partial D}^{(n)}(\tilde{x})<\boldsymbol{\delta} \boldsymbol{t}\right] \rightarrow \exp \left(-\nu h\left(x^{\perp}\right)\right),
$$


where $h\left(\tilde{x}^{\perp}\right)$ is the distance from $\tilde{x}=\left(\tilde{x}^{\perp}, 0\right)$ to $\partial D$. Taking $\nu s=\mathcal{O}(1)$ and using (3.9), we can expand the exponential as follows:

$$
\exp \left(-\nu h\left(x^{\perp}\right)\right)=1-\frac{1}{2} \nu\left(\kappa_{1} x_{1}^{2}+\kappa_{2} x_{2}^{2}+\cdots+\kappa_{n-1} x_{n-1}^{2}\right)+\mathcal{O}\left(\left(\frac{\kappa}{\nu}\right)^{2}\right) .
$$

Thus, as $\kappa / \nu \rightarrow 0$ with $\nu s$ fixed,

$$
\mathcal{P}\left[\mathbf{H}_{\partial D}^{(n)}(x)<\boldsymbol{\delta} \boldsymbol{t}\right]=\mathrm{e}^{-\nu s}\left(1-\frac{1}{2} s\left(\kappa_{1}+\kappa_{2}+\cdots+\kappa_{n-1}\right)+\mathcal{O}\left(\left(\frac{\kappa}{\nu}\right)^{2}\right)\right) .
$$

A similar argument can be used to show that (3.15) holds when $\kappa_{i}<0$.

For the boundary test, we need conditional hitting probabilities, given the end point of a timestep. Let

$$
c\left(x, x^{\prime}\right)=\mathcal{P}\left[\mathbf{H}_{\partial D}^{(n)}(x)<\boldsymbol{\delta} \boldsymbol{t} \mid \mathbf{B}_{\boldsymbol{\delta} t}=x^{\prime}\right] .
$$

Because of the exponential distribution of $\boldsymbol{\delta} \boldsymbol{t}$, any one path can be considered as starting afresh at the boundary and the conditional probability can be written as the product

$$
c\left(x, x^{\prime}\right)=\mathcal{P}\left[\mathbf{H}_{\partial D}^{(n)}(x)<\boldsymbol{\delta} \boldsymbol{t}\right]\left(p_{\nu}^{(n)}\left(x^{\prime}-x\right)\right)^{-1} p_{\nu}^{*}\left(x, x^{\prime}\right),
$$

where $p_{\nu}^{(n)}(x)$ is given by $(2.2)$,

$$
p_{\nu}^{*}\left(x, x^{\prime}\right)=\int p_{\nu}^{(n)}\left(x^{\prime}-y\right) q_{\partial D}\left(y^{\perp}\right) \mathrm{d} y^{\perp}
$$

and $q_{\partial D}\left(y^{\perp}\right)$ is the density of arrival positions on $\partial D$, defined in the same way as (3.4). In one dimension, $p_{\nu}^{*}\left(x, x^{\prime}\right)$ is the density at $x^{\prime}$ after an exponential timestep started at the boundary and is independent of $x$. In more than one dimension, $p_{\nu}^{*}\left(x, x^{\prime}\right)$ is the density at $x^{\prime}$ resulting from the ensemble of realizations with initial conditions distributed according to the density of arrival positions on $\partial D$, which depends on $x$. Analysis of the conditional hitting probability (3.17) is the basis for boundary tests of arbitrary accuracy.

4. Exponential timestepping. In this section, we describe the update algorithm and alternative boundary test criteria that we have implemented for exponential timestepping in $n$ dimensions. The Cartesian components of the increment of an $n$ dimensional Brownian motion over a time interval of fixed length are independent Gaussian random variables. Under exponential timestepping, the Cartesian coordinates of the increment are no longer independent. However, the fact that the distance traveled is independent of the direction traveled permits a simple algorithm to be developed.

4.1. Multidimensional increments. The density of the distance traveled after an exponentially distributed time is obtained from (2.9) by letting $r_{0} \rightarrow 0$, which gives

$$
b^{(n)}(r, 0)=2^{1-n / 2} \nu(\nu r)^{n / 2} K_{n / 2-1}(\nu r) / \Gamma(n / 2) .
$$

To carry out an exact update for Brownian motion in $n$ dimensions, we generate an increment whose modulus is $\nu^{-1} \mathbf{a}^{(n)}$, where

$$
\frac{\mathrm{d}}{\mathrm{d} x} \mathcal{P}\left[\mathbf{a}^{(n)}<x\right]=2^{1-n / 2} x^{n / 2} K_{n / 2-1}(x) / \Gamma\left(\frac{n}{2}\right) .
$$


We construct realizations of the random variable $\mathbf{a}^{(n)}$ as

$$
\mathbf{a}^{(n)}=\left(\mathbf{h}_{1}^{2}+\mathbf{h}_{2}^{2}\right)^{1 / 2}\left(\mathbf{g}_{1}^{2}+\mathbf{g}_{2}^{2}+\cdots+\mathbf{g}_{n}^{2}\right)^{1 / 2},
$$

where $\mathbf{h}_{1}, \mathbf{h}_{2}$, and $\mathbf{g}_{1}, \mathbf{g}_{2}, \ldots, \mathbf{g}_{n}$ are independent Gaussian random variables with zero mean and unit variance [24]. The factor $\left(\mathbf{h}_{1}^{2}+\mathbf{h}_{2}^{2}\right)^{1 / 2}$ is conveniently generated as $(-2 \log \mathbf{u})^{1 / 2}$, where $\mathbf{u}$ is distributed uniformly on $(0,1)[25]$.

The direction of the increment is independent of the modulus and uniformly distributed on the surface of the unit $n$-dimensional ball. Let $\mathbf{v}_{1}$ and $\mathbf{v}_{2}$ be independent and uniformly distributed between -1 and 1 . For all pairs such that $\mathbf{v}_{1}^{2}+\mathbf{v}_{2}^{2}<1$, let $\mathbf{x}=\left(\mathbf{v}_{1}^{2}-\mathbf{v}_{2}^{2}\right) /\left(\mathbf{v}_{1}^{2}+\mathbf{v}_{2}^{2}\right)$ and $\mathbf{y}=2 \mathbf{v}_{1} \mathbf{v}_{2} /\left(\mathbf{v}_{1}^{2}+\mathbf{v}_{2}^{2}\right)$. Then $(\mathbf{x}, \mathbf{y})$ is uniformly distributed on the unit circle [26]. A uniform distribution on the unit sphere is produced by choosing $\mathbf{z}$ uniformly in $(-1,1)$; then $\mathbf{x}$ and $\mathbf{y}$ uniformly on a circle with radius $\left(1-\mathbf{z}^{2}\right)^{1 / 2}$. In dimension $n>3$, two possible methods are as follows. First, a vector whose $n$ components are independent mean-zero Gaussian random variables with equal variance has density that depends only on its magnitude. Normalizing to unit magnitude produces a uniform distribution on the $n$-dimensional sphere. Alternatively, for $n$ not much above 3, one may sample from a uniform distribution in the $n$-cube that contains the unit ball, reject those outside the ball, then renormalize to unit magnitude.

4.2. Boundary tests. A boundary test is performed after each update of the process to take account of the probability that the boundary has been hit during the timestep. The boundary is deemed to have been hit the first time that

$$
C_{\text {num }}\left(s, s^{\prime}\right)>\mathbf{v}
$$

where $C_{\text {num }}\left(s, s^{\prime}\right)$ is the approximation of (4.7) used in the numerical method and $\mathbf{v}$ is drawn, independently at each timestep, from a uniform distribution on $(0,1)$.

The full information available for the boundary test is the value of the process at the start of the timestep, $x$, and the value at the end, $x^{\prime}$ (Figure 4.1). However, the conditional probability,

$$
\mathcal{P}\left[\mathbf{H}_{P}^{(n)}(x)<\boldsymbol{\delta} \boldsymbol{t} \mid \mathbf{B}_{\boldsymbol{\delta} \boldsymbol{t}}=x^{\prime}\right]=\exp \left(-2 \nu\left(s \wedge s^{\prime}\right)\right),
$$

is exact for the flat surface $P$, even though it makes no use of the information contained in the directions parallel to $P$. It can be obtained from (3.17) by integrating over $x^{\perp}$ and $\left(x^{\prime}\right)^{\perp}$. We call a boundary test using (4.5) the simple boundary test. For numerical timesteps of fixed length $\Delta t$, the flat boundary approximation has been called a "half-space" approximation in [15]. The corresponding Euler boundary test is

$$
C_{\text {Euler }}\left(s, s^{\prime}\right)=\exp \left(2 s s^{\prime} \Delta t^{-1}\right) .
$$

We seek corrections to (4.5) by analyzing the conditional hitting probability in the form

$$
\bar{c}\left(s, s^{\prime}\right)=\mathcal{P}\left[\mathbf{H}_{\partial D}^{(n)}(x)<\boldsymbol{\delta} \boldsymbol{t} \mid x_{n}^{\prime}=-s^{\prime}\right] .
$$

This is a simplification of (3.16) because the conditional hitting probability is analyzed as a function of $s \in \mathbb{R}$ and $s^{\prime} \in \mathbb{R}$, rather than as a function of $x \in \mathbb{R}^{n}$ and $x^{\prime} \in \mathbb{R}^{n}$. We also need only the properties of the surface at its point nearest $x$. 


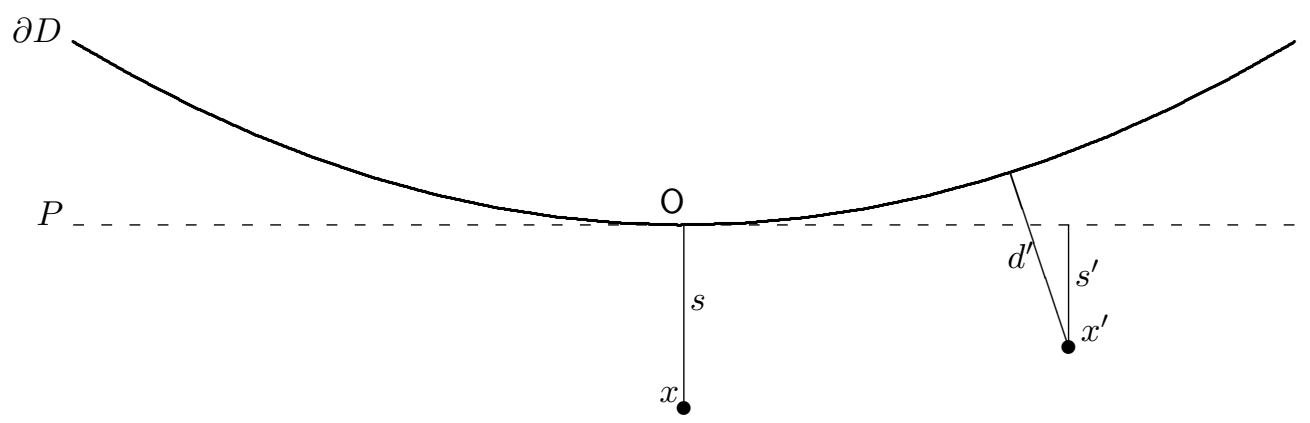

FIG. 4.1. The position at the start of a timestep is $x$ and the position at the end of the timestep is $x^{\prime}$. The distance from $x$ to $\partial D$ is $d$ and the distance from $x^{\prime}$ to $\partial D$ is $d^{\prime}$. The distance from $x$ to $P$ is $s=d$ and the distance from $x^{\prime}$ to $P$ is $s^{\prime} \neq d^{\prime}$.

Assuming that the surface $\partial D$ can be parametrized as in (3.12), (4.7) is

$$
\begin{aligned}
\bar{c}\left(s, s^{\prime}\right) & =\mathcal{P}\left[\mathbf{H}_{\partial D}^{(n)}(x)<\boldsymbol{\delta} \boldsymbol{t}\right]\left(p_{\nu}^{(1)}\left(s-s^{\prime}\right)\right)^{-1} \int p_{\nu}^{(1)}\left(y_{n}\left(y^{\perp}\right)+s^{\prime}\right) q_{\partial D}\left(y^{\perp}\right) \mathrm{d} y^{\perp} \\
& =\mathcal{P}\left[\mathbf{H}_{\partial D}^{(n)}(x)<\boldsymbol{\delta} \boldsymbol{t}\right] \exp \left(\nu\left|s^{\prime}-s\right|-\nu s^{\prime}\right) \int \exp \left(-\nu y_{n}\left(y^{\perp}\right)\right) q_{\partial D}\left(y^{\perp}\right) \mathrm{d} y^{\perp}
\end{aligned}
$$

Note that, if all $\kappa_{i}$ in (3.12) are zero, then $y_{n}\left(y^{\perp}\right)=0$ and (4.8) reduces to (4.5). The first factor in (4.8) was evaluated in (3.15). Now, similarly to (3.14),

$$
\exp \left(-\nu y_{n}\left(x^{\perp}\right)\right)=1-\frac{1}{2} \nu\left(\kappa_{1} y_{1}^{2}+\kappa_{2} y_{2}^{2}+\cdots+\kappa_{n-1} y_{n-1}^{2}\right)+\mathcal{O}\left(\left(\frac{\kappa}{\nu}\right)^{2}\right)
$$

where $y_{i}=\mathcal{O}\left(\nu^{-1}\right)$. With (3.12) we find

$$
\begin{aligned}
\int \exp \left(-\nu y_{n}\left(x^{\perp}\right)\right) q_{\partial D}\left(x^{\perp}\right) \mathrm{d} x^{\perp}= & 1-\frac{1}{2} s\left(\kappa_{1}+\kappa_{2}+\cdots+\kappa_{n-1}\right) \\
& +\mathcal{O}\left(\left(\frac{\kappa}{\nu}\right)^{2}\right) .
\end{aligned}
$$

Using (3.15) and (4.10) in (4.8) we find the curvature-corrected boundary test, with errors proportional to $(\kappa / \nu)^{2}$ :

$$
C_{\mathrm{cc}}\left(s, s^{\prime}\right)=\exp \left(-2 \nu\left(\left(s \wedge s^{\prime}\right) \vee 0\right)\right)\left(1-s\left(\kappa_{1}+\kappa_{2}+\cdots+\kappa_{n-1}\right)\right) .
$$

We also implement a symmetric boundary test, invariant under interchange of the start and end points of the timestep,

$$
C_{\mathrm{sym}}=\exp \left(-2 \nu\left(d \wedge d^{\prime}\right)\right)
$$

An extra complication arises when measuring the exit time from a nonconcave domain. In that case $\partial D$ may be reached before $P$. If $x^{\prime}$ is between $\partial D$ and $P$, then the boundary is deemed hit; the conditional hitting probability (4.4) needed for the boundary test is that restricted to those paths with $x^{\prime}$ inside the domain. Consider the conditional hitting probability as a function of $s^{\prime}$ for a given $s$. As $s^{\prime} \rightarrow \infty$, we regain 
(4.11). On the other hand, as $s^{\prime} \rightarrow 0$, the curvature is not seen by the subset of paths that remain inside the domain, and so the limit is (4.5). In our numerical experiments with exit from domains such that $\kappa_{i}<0$ for some $i$, we use the curvature-corrected convex boundary test given by

$$
C_{\mathrm{cv}}\left(s, s^{\prime}\right)=\exp \left(-2 \nu\left(s \wedge s^{\prime}\right)\right)\left(1-\left(s \wedge s^{\prime}\right)\left(\kappa_{1}+\kappa_{2}+\cdots+\kappa_{n-1}\right)\right) .
$$

5. Numerical experiments. We restrict ourselves to Brownian motion, where the update is exact under both Euler and exponential timestepping, but a systematic error in the numerical mean exit time may arise from the numerical decision as to when the boundary is deemed to have been hit. Simple use of a boundary test gives an error in the mean exit time proportional to $\lambda^{-1}$, the mean duration of a timestep [2]. In this work we are able to obtain high-order convergence of the mean exit time under exponential timestepping.

We begin with exits from circles and spheres, where the problem can be transformed into a one-dimensional problem (although the transformed process is no longer driftless). In the case of an exit from a circle of radius $R$, the exact conditional hitting probability involves special functions that can be expanded in powers of $(\nu R)^{-1}$ in a form convenient for numerical implementation. The more terms that are included in the expansion, the higher is the order of the numerical method. In the case of exit from a sphere, the special functions reduce to a simple form that can be used to produce numerical estimates of the mean exit time with no systematic error.

We also study exit from the interior of an ellipse and from the region bounded by the two lines of a hyperbola. These examples illustrate the two cases of locally convex and locally concave domains with smooth boundaries that could be found in applications, and have the advantage that exact analytical results are available for comparison with numerics.

Numerical results are plotted as a function of the parameter $\lambda^{-1}$, the mean duration of a timestep. The same horizontal axis is used to display results obtained using the Euler method, where the duration of a timestep, $\Delta t$, is fixed and equal to $\lambda^{-1}$. We choose $D=\frac{1}{2}$ in all numerical experiments, which typically consist of $10^{10}$ realizations.

The programs used in the numerical experiments reported in this section are available at http://randomideas.org.

5.1. Exits from circles and spheres. We consider the problem of the numerical estimation of the mean exit time from an $n$-dimensional ball. The mean exit time with initial condition $x \in \mathbb{R}^{n}$ is denoted by $\left\langle\mathbf{h}_{R}^{(n)}(x)\right\rangle$ and given by

$$
\left\langle\mathbf{h}_{R}^{(n)}(x)\right\rangle=\frac{R^{2}-|x|^{2}}{n} .
$$

Figure 5.1 was obtained using the naive method of evaluating a mean exit time; i.e., each realization is continued without a boundary test until the first time that a value is generated at a distance greater than $R$ from the origin. The difference between the mean time recorded and (5.1) is proportional to $\langle\boldsymbol{\delta} \boldsymbol{t}\rangle^{1 / 2}$.

In Figure 5.2, we show the error in the mean exit time from a circle using various boundary tests and timestepping methods. The results labeled "Euler" are obtained using the Euler update and the boundary test (4.6). The results labeled "simple" and "corrected" are obtained using exponential timestepping and boundary tests based on (2.13). When only the leading term in (2.13) is used, we obtain errors proportional 
Exit from circle

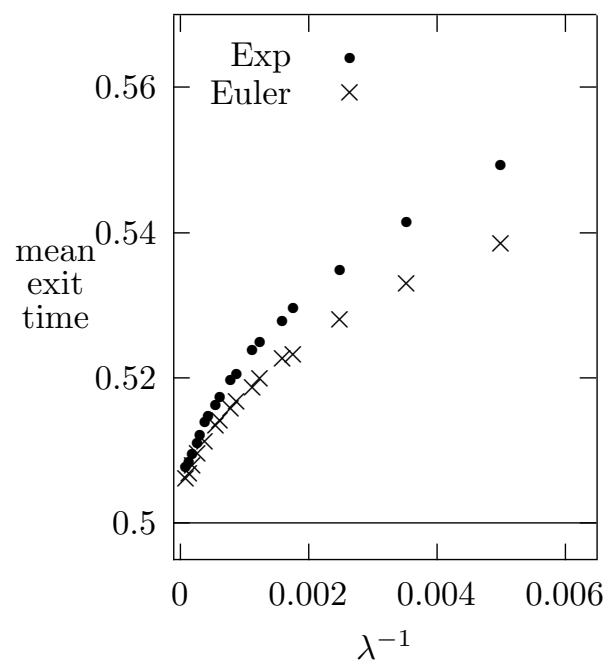

Exit from sphere

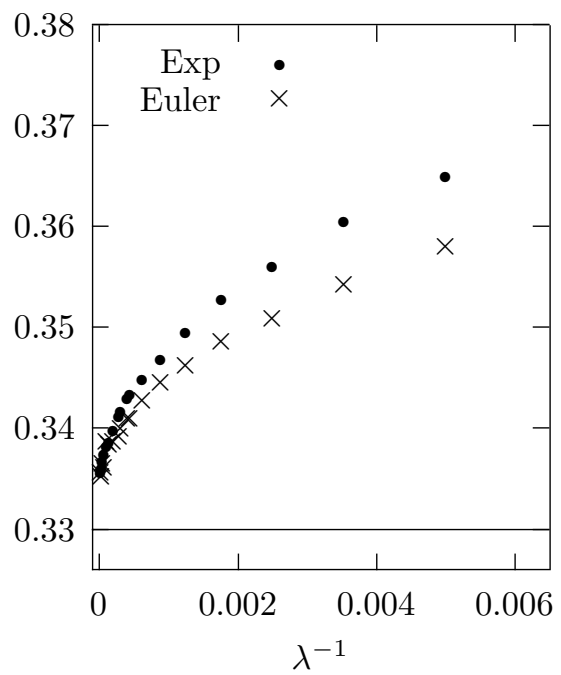

FIG. 5.1. Measuring the mean exit time without a boundary test. Exit from a circle (left) and sphere (right) with unit radius, starting from the origin. Although the updates of the process are exact, the mean exit time is overestimated by an amount proportional to the square root of the mean duration of a timestep. The horizontal axis is the mean duration of a timestep in the case of exponential timestepping and the (fixed) length $\Delta t$ of a timestep in the case of the Euler method. Statistical errors are smaller than the plotted symbols.

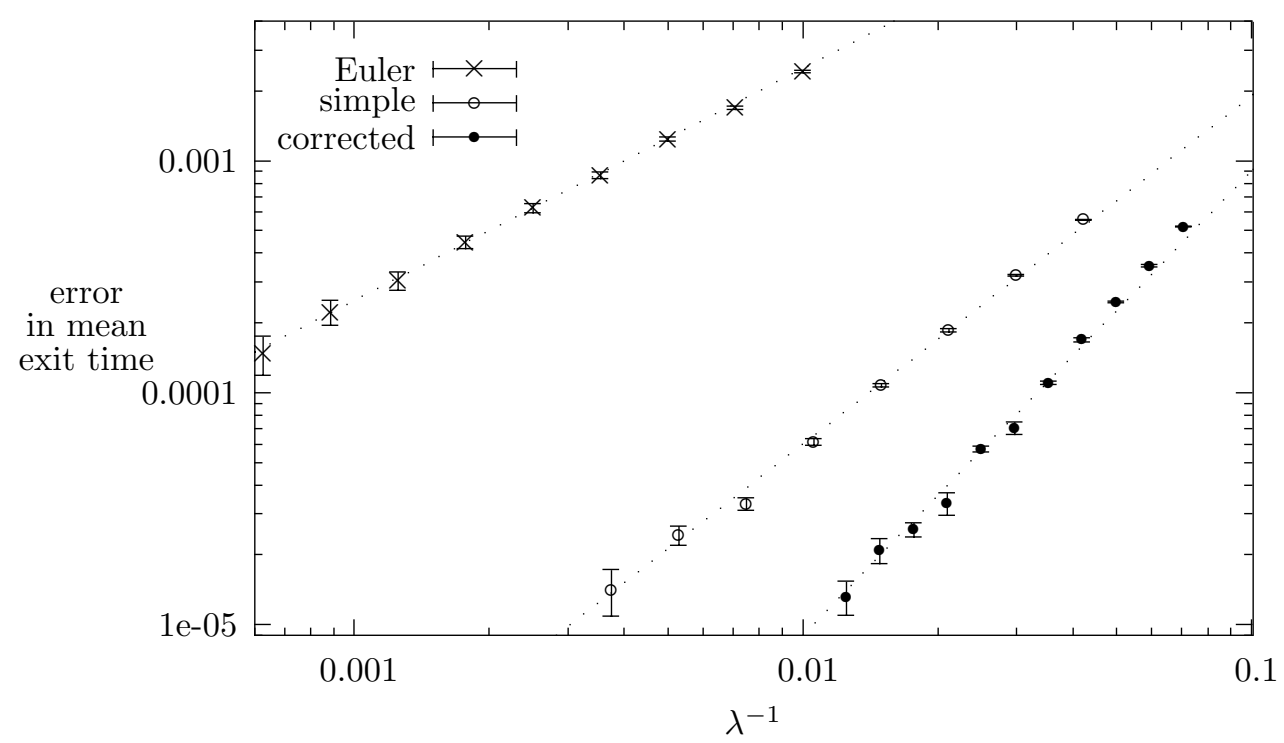

FIG. 5.2. Error in numerical mean exit time, using boundary correction (logarithmic scales). Exit from a circle with radius $R=1$, starting from the origin. Statistical errors are indicated by error bars. The dotted lines are error $=0.25\langle\boldsymbol{\delta} \boldsymbol{t}\rangle$, error $=0.06\langle\boldsymbol{\delta} \boldsymbol{t}\rangle^{3 / 2}$, and error $=0.10\langle\boldsymbol{\delta} \boldsymbol{t}\rangle^{2}$. 


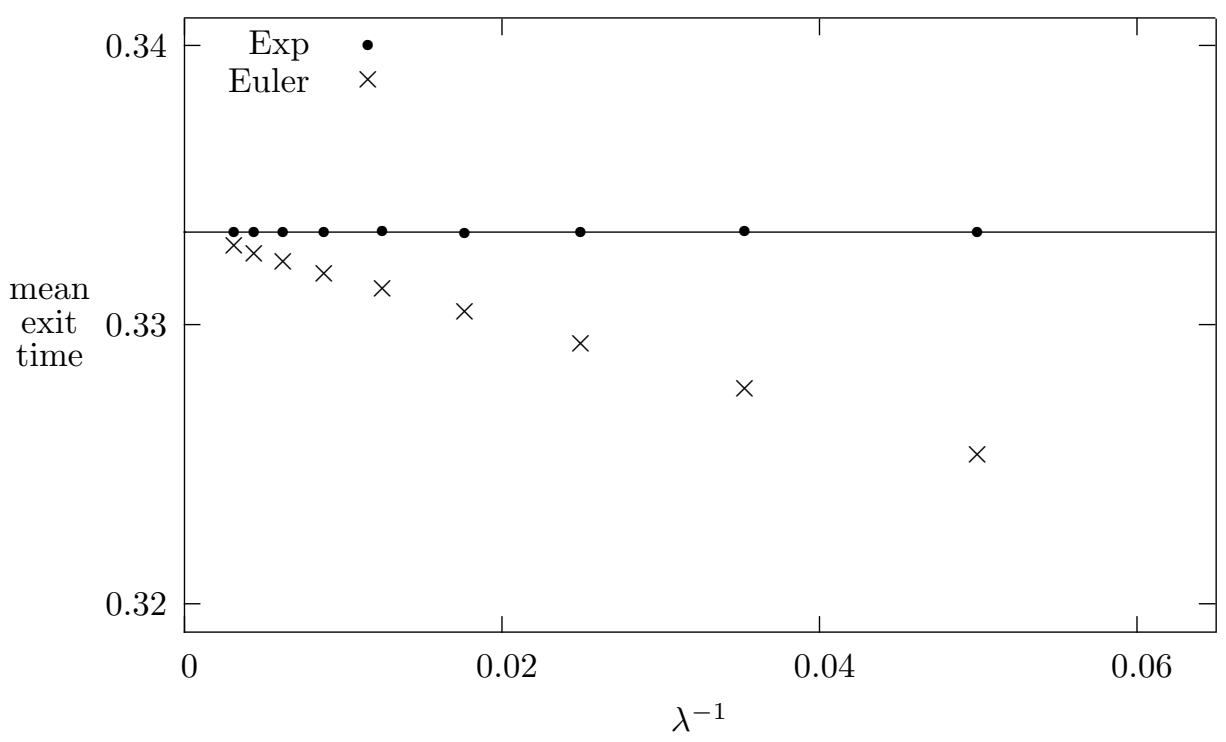

FIG. 5.3. Error in numerical mean exit time, using boundary correction. Exit from a sphere with radius $R=1$, starting from the origin. The solid line is the exact result (5.1). The systematic error is zero for the exponential method with boundary test. Statistical errors are smaller than the plotted symbols.

to $\langle\boldsymbol{\delta} \boldsymbol{t}\rangle^{3 / 2}$. When the next term in (2.13) is included, we find errors proportional to $\langle\boldsymbol{\delta} \boldsymbol{t}\rangle^{2}$. The size of the statistical errors is independent of $\lambda$, but on a logarithmic scale they appear large when the systematic error is small.

When $n=3$, we find the convenient exact form

$$
\mathcal{P}\left[\mathbf{h}_{R}^{(3)}\left(r_{0}\right)<\boldsymbol{\delta} \boldsymbol{t}|| \mathbf{B}_{\boldsymbol{\delta} \boldsymbol{t}} \mid=r_{1}\right]=\mathrm{e}^{-\nu|R-r|} \frac{\sinh \nu(r \vee R)}{\sinh \nu(r \wedge R)},
$$

where $r$ is the closer of $r_{0}$ and $r_{1}$ to $R$. The mean exit time from a sphere is obtained without systematic error for any value of $\lambda$ under exponential timestepping. In Figure 5.3 the mean exit time is plotted against $\lambda^{-1}$. Results obtained using exponential timestepping with (5.2) are shown as small filled circles and results obtained using the Euler method with (4.6) as crosses.

5.2. Exit from an ellipse. As an example of a convex domain, we consider the interior of an ellipse, i.e., the set of points $\left(x_{1}, x_{2}\right) \in \mathbb{R}^{2}$ satisfying

$$
a x_{1}^{2}+b x_{2}^{2}<1,
$$

where $a$ and $b$ are positive constants. Let $\mathbf{H}_{a, b}\left(x_{1}, x_{2}\right)$ be the first time that a Brownian motion, started at $\left(x_{1}, x_{2}\right)$, exits the interior of the ellipse. Then $\left\langle\mathbf{H}_{a, b}\left(x_{1}, x_{2}\right)\right\rangle=$ $T\left(x_{1}, x_{2}\right)$, where

$$
\frac{1}{2}\left(\frac{\partial^{2}}{\partial x_{1}^{2}}+\frac{\partial^{2}}{\partial x_{2}^{2}}\right) T\left(x_{1}, x_{2}\right)=-1, \quad T\left(x_{1}, x_{2}\right)=0 \quad \text { when } \quad a x_{1}^{2}+b x_{2}^{2}=1 .
$$




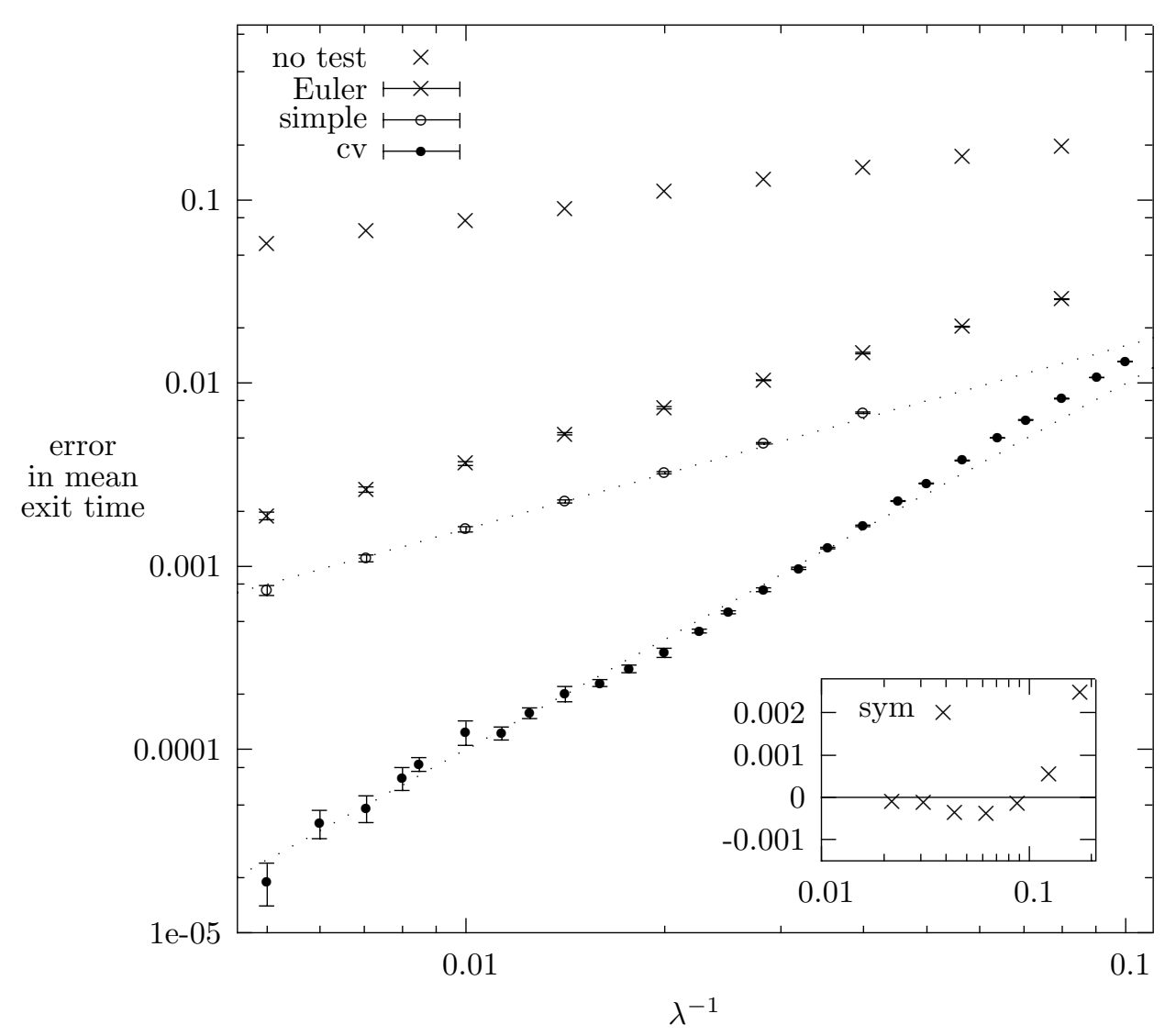

FIG. 5.4. Error in numerical mean exit time (logarithmic scales). Exit from an ellipse, described by (5.3) with $a=1 / 4$ and $b=1$. The crosses are results using the Euler method, with no boundary test (crosses) and with the boundary test (4.6) (crosses with error bars). The results labeled "simple" were obtained using the boundary test, (4.5); those labeled "cv" were obtained using the curvature-corrected convex boundary test, (4.13). The dotted lines are error $=0.16\langle\boldsymbol{\delta} \boldsymbol{t}\rangle^{1}$ and error $=1.5\langle\boldsymbol{\delta} \boldsymbol{t}\rangle^{2}$. The inset displays the difference between the numerical and exact mean exit times using the symmetric boundary test (4.12).

The solution of (5.4) is

$$
T\left(x_{1}, x_{2}\right)=\left(1-a x_{1}^{2}-b x_{2}^{2}\right) /(a+b) .
$$

In our numerical experiments, the update is the same as that used for studying exits from circles. The boundary is deemed hit if the end point of a timestep is outside the ellipse or if the boundary test is satisfied. To evaluate the conditional probabilities for the boundary test, the closest point on the ellipse to the starting point of each timestep is found by Newton iteration.

In Figure 5.4, we display results obtained using five different methods: the standard Euler method with no boundary test and with the boundary test (4.6), and exponential timestepping with the boundary tests (4.5), (4.13), and (4.12). With (4.5) we find a systematic error in the mean exit time proportional to $\langle\boldsymbol{\delta} \boldsymbol{t}\rangle^{1}$; with (4.13) we find a systematic error proportional to $\langle\boldsymbol{\delta} \boldsymbol{t}\rangle^{2}$. Using (4.12) produces an error that passes through 0 at a finite value of $\lambda$ (inset). 

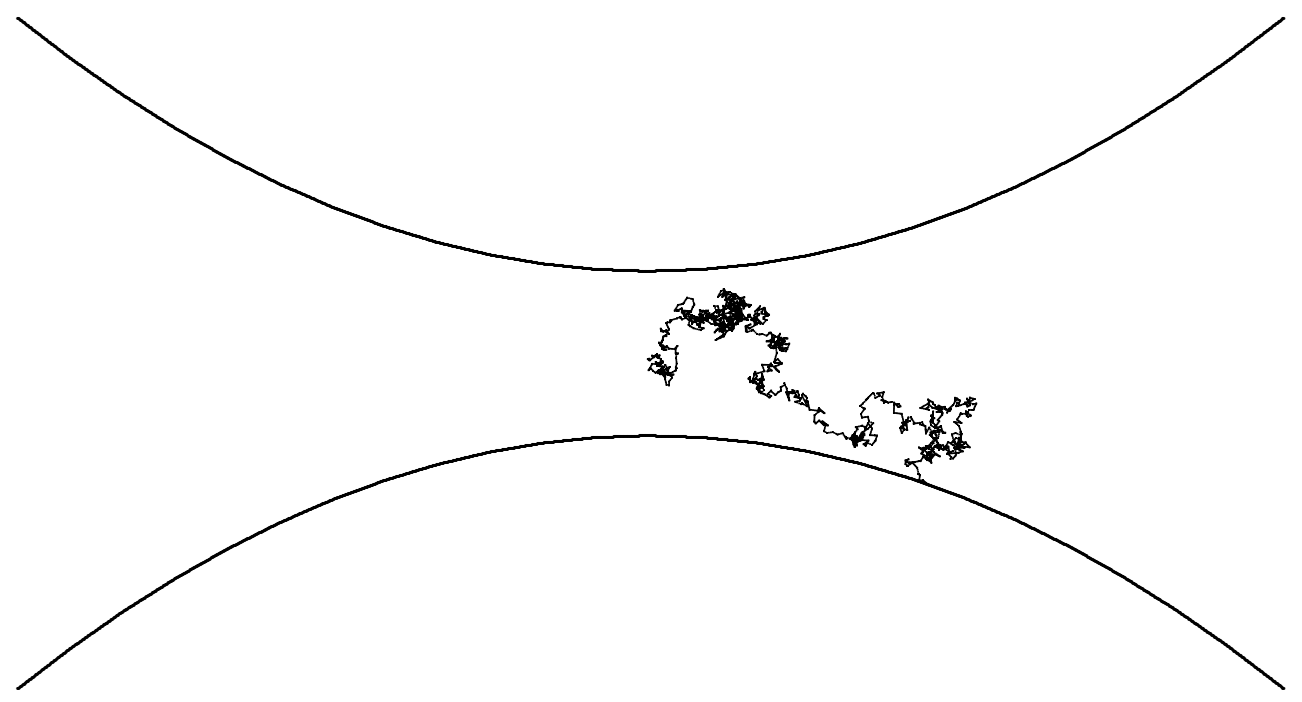

FIG. 5.5. We measure the mean exit time from concave regions bounded by the two lines of a hyperbola. The figure shows one realization.

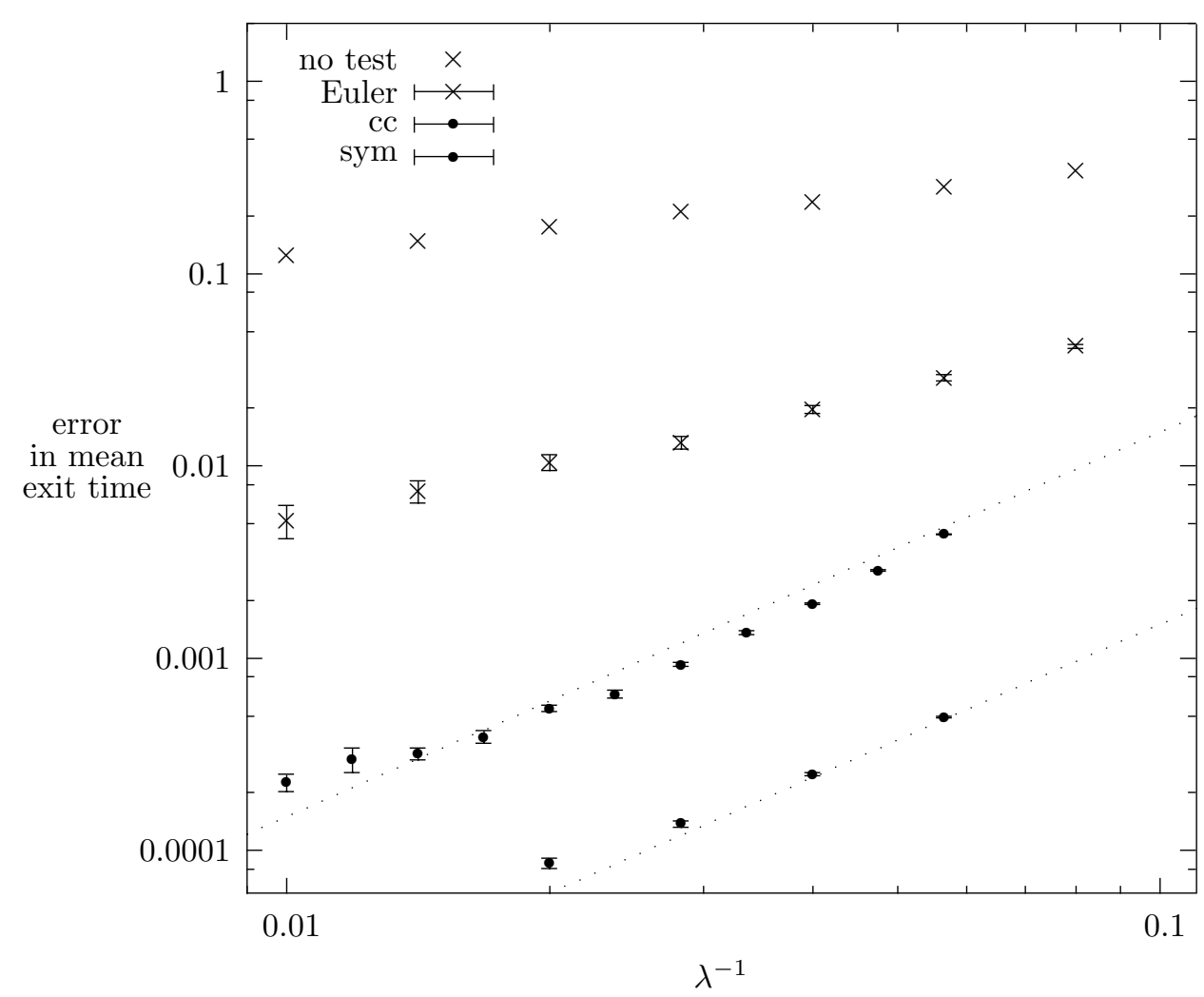

FIG. 5.6. Error in numerical mean exit time, using boundary correction (logarithmic scales). Exit from a two-dimensional concave region. The hyperbola is described by (5.3) with $a=-1 / 16$ and $b=1$. The dotted lines are error $=1.5\langle\boldsymbol{\delta} \boldsymbol{t}\rangle^{2}$ and error $=0.15\langle\boldsymbol{\delta} \boldsymbol{t}\rangle^{2}$. 
5.3. Exit from a region bounded by the two lines of a hyperbola. Consider a hyperbola, defined as the set of points $\left(x_{1}, x_{2}\right) \in \mathbb{R}^{2}$ satisfying

$$
a x_{1}^{2}+b x_{2}^{2}=1,
$$

where $a<0$ and $b>0$. The mean exit time from the concave region between the two lines of the hyperbola, depicted in Figure 5.5, is also given by (5.5).

The numerical runs whose results are displayed in Figure 5.6 were performed using the symmetric boundary test (4.12) and the curvature-corrected boundary test (4.11). Statistical errors are large because many paths make long excursions before reaching a boundary. Both boundary tests used produce a systematic error proportional to $\langle\boldsymbol{\delta} \boldsymbol{t}\rangle^{2}$. The symmetric boundary test is the most efficient for this concave region; the phenomenon of change of sign of the error at a finite value of $\lambda$, seen in the ellipsoidal region studied in section 5.2, is not found.

6. Conclusion. An exact numerical procedure has been described for the update of Brownian motion in $n$ dimensions under exponential timestepping. The modulus of an increment is generated as a combination of simple random variables and the angle is uniformly distributed on the surface of the $n$-dimensional ball.

To evaluate a mean exit time, numerous numerical realizations are generated, each proceeding by updating the process, then performing a test for the possibility of a boundary having been reached in the time interval. A realization is brought to an end when the boundary is deemed hit. Under exponential timestepping, numerical orders of convergence greater than 1 are attainable because of the simple relationship that holds between the mean number of timesteps per realization and the mean exit time. Numerical evidence is presented for convergence proportional to the square of the mean duration of a timestep. An analytical demonstration that this is indeed the case will be the subject of future research.

Taking as examples the exit times from circles, spheres, ellipses, and regions bounded by the two lines of a hyperbola, we report the results of numerical experiments performed with the algorithms developed in this work. The mean exit time from a circle of radius $R$ can be evaluated to arbitrary accuracy by expanding the conditional hitting probability in powers of $(\nu R)^{-1}$. The mean exit time from a sphere is evaluated without systematic error.

The simple boundary test for general smooth surfaces under exponential timestepping uses the minimum of the distance from the starting point and the ending point to the tangent plane through the point on the boundary closest to the starting point. We were able to obtain second-order convergence, i.e., an error proportional to the square of the mean duration of a timestep, using a boundary test that includes a term proportional to $\kappa / \nu$, where $\kappa$ is the curvature of the boundary measured at the point on the boundary closest to the starting point. Still higher orders of convergence are attainable if boundary tests including further terms in (3.12) can be derived.

We have considered concave and convex smooth boundaries. Analysis of hitting times of hyperbolae may prove useful in devising algorithms to deal with regions with corners because a suitable choice of parameters gives a geometry approximating that found near a corner with an acute angle. Exponential timestepping has been implemented for processes other than Brownian motion in one dimension [14]. The best way to generalize to multiple dimensions may be to use an approximation by mixing two processes with constant drifts at each timestep. Other areas for future development are algorithms capable of dealing with several close boundaries simultaneously, and boundary tests in three-dimensional regions that take as their starting point the simple exact solution for the conditional hitting probability of a sphere. 


\section{REFERENCES}

[1] P. E. Klofden and E. Platen, Numerical Solution of Stochastic Differential Equations, Springer, Berlin, 1992.

[2] K. M. Jansons AND G. D. Lythe, Efficient numerical solution of stochastic differential equations using exponential timestepping, J. Statist. Phys., 100 (2000), pp. 1097-1109.

[3] K. Itô And H. P. McKean, JR., Diffusion Processes and Their Sample Paths, Springer, Berlin, 1974.

[4] Z. Schuss, Theory and Applications of Stochastic Differential Equations, Wiley, New York, 1980.

[5] S. Karlin and H. M. Taylor, A Second Course in Stochastic Processes, Academic Press, Orlando, FL, 1981.

[6] C. W. Gardiner, Handbook of Stochastic Methods, Springer, Berlin, 1985.

[7] I. Karatzas And S. E. Shreve, Brownian Motion and Stochastic Calculus, Springer, New York, 1988.

[8] S. Redner, A Guide to First-Passage Processes, Cambridge University Press, Cambridge, UK, 2001.

[9] M. Beccaria, G. Curci, and A. Viceré, Numerical solutions of first-exit-time problems, Phys. Rev. E, 48 (1993), pp. 1539-1546.

[10] P. BALDI, Exact asymptotics for the probability of exit from a domain and applications to simulation, Ann. Probab., 23 (1995), pp. 1644-1670.

[11] G. N. Milstein and M. V. Tretyakov, Simulation of a space-time bounded diffusion, Ann. Appl. Probab., 9 (1999), pp. 732-779.

[12] R. Mannella, Boundaries and optimal stopping in a stochastic differential equation, Phys. Lett. A, 254 (1999), pp. 257-262.

[13] E. Gobet, Weak approximation of killed diffusion using Euler schemes, Stochastic Process. Appl., 87 (2000), pp. 167-197.

[14] K. M. Jansons And G. D. Lythe, Exponential timestepping with boundary test for stochastic differential equations, SIAM J. Sci. Comput., 24 (2002), pp. 1809-1822.

[15] E. Gobet, Euler schemes and half-space approximation for the simulation of diffusion in a domain, ESAIM Probab. Stat., 5 (2001), pp. 261-297.

[16] M. E. Muller, Some continuous Monte Carlo methods for the Dirichlet problem, Ann. Math. Statist., 27 (1956), pp. 569-589.

[17] C.-O. Hwang, M. Mascagni, and J. A. Given, A Feynman-Kac path-integral implementation for Poisson's equation using an h-conditioned Green's function, Math. Comput. Simulation, 62 (2003) pp. 347-355.

[18] F. M. Buchmann, Simulation of stopped diffusions, J. Comput. Phys., 202 (2005), pp. 446-462.

[19] A. N. Borodin And P. Salminen, Handbook of Brownian Motion-Facts and Formulae, Birkhäuser, Basel, 1996.

[20] K. M. Jansons And G. D. Lythe, Stochastic calculus: Application to dynamic bifurcations and threshold crossings, J. Statist. Phys., 90 (1998), pp. 227-251.

[21] L. A. Santaló, Integral Geometry and Geometric Probability, Encyclopedia Math. Appl. 1, Addison-Wesley, Reading, MA, 1976.

[22] C. G. Phillips And K. M. Jansons, The short-time transient of diffusion outside a conducting body, Proc. Roy. Soc. London Ser. A, 428 (1990), pp. 431-449.

[23] F. Knight, Essentials of Brownian Motion and Diffusion, AMS, Providence, RI, 1981.

[24] K. S. Miller, Multidimensional Gaussian Distributions, Wiley, New York, 1964.

[25] B. D. Ripley, Stochastic Simulation, Wiley, New York, 1987.

[26] D. E. Knuth, Seminumerical Algorithms, 3rd Ed., Addison-Wesley, Reading, MA, 1998. 